\title{
Edge Computing: Utilization of the Internet of Things for Time- Sensitive Data Processing
}

\author{
Apoorva Ganapathy \\ Senior Developer, Adobe Systems, San Jose, California, USA \\ *E-mail for correspondence: apganapa@adobe.com \\ https://doi.org/10.18034/abr.v11i2.547
}

\begin{abstract}
An edge computing system is a shared IT (Information Technology) system where customer data can be processed at the edge of the network to as close as possible to the originating source. The Internet of Things connects the various things on the internet, making it easier to live and allow jobs to be done more smartly. It also gives total control to the users. The combination of Edge computing and the Internet of Things can potentially result in huge possibilities for users. This work accessed edge computing and the benefits of using edge computing. It also looked into how to edge the many possibilities that can result in the use of edge computing. Various similar concepts like fog and cloud computing were also considered as closely related terms. This article provides insights into the use of edge computing in several industries.
\end{abstract}

Key words: Edge-computing, Internet of Things, Artificial Intelligence, Cloud, Bandwidth, End-Point, Machine Learning

\section{INTRODUCTION}

The growth and development of new technologies have greatly affected the way tasks are carried out. Edge computing and the Internet of Things (IoT) are two innovations of modern age technological advancement. There is a constant increase in the number of things connected to the internet of things and the volume of data produced by these devices. This increase can have negative effects on the IoT. Edge computing is an information technology system that allows data processing at the source where the data was created. Edge computing is a way to bypass the latency caused by cloud computing and getting data to a data center for processing. Edge computing can process time-sensitive data in remote locations with limited or no connectivity to a centralized location. In those situations, edge computing can act like mini datacenters.

\section{THE INTERNET OF THINGS}

The IoT network comprises systems and a network of web-connected intelligent devices that employ encoded networks like sensors, processors, and interactive hardware to receive, send and store data. The network in the IoT uses the stored data. To use the stored data, the
IoT device sends out the collected sensor information through a connection with a gateway on the IoT or through other means such as using devices with edge tools where data is either sent for analysis on the local network or in the cloud. Connected devices on the IoT network interact with each other and use the shared data they acquire to solve problems. The interactions within the network are done without human supervision or interference, and most of the job is carried out by the devices. Even though humans may communicate with the devices to set them up, writing scripts for protocols and commands provides access to information.

The Different applications used by IoT decide the protocol and system of connection, communication, and network which the things or devices are to use. Artificial Intelligence (AI) and machine learning in IoT improve data collection and processing by the connected devices in a network system. Machine learning makes it dynamic.

The Internet of Things connects the various things on the internet, making it easier to live and allow jobs to be done more smartly. It also gives total control to the users.

Also, for business enterprises, IoT gives real-time analysis of their systems' performance and provides examinations 
into everything regarding the functions of the devices in the supply network and logistics (Azad et al., 2021). This makes IoT important for enterprises.

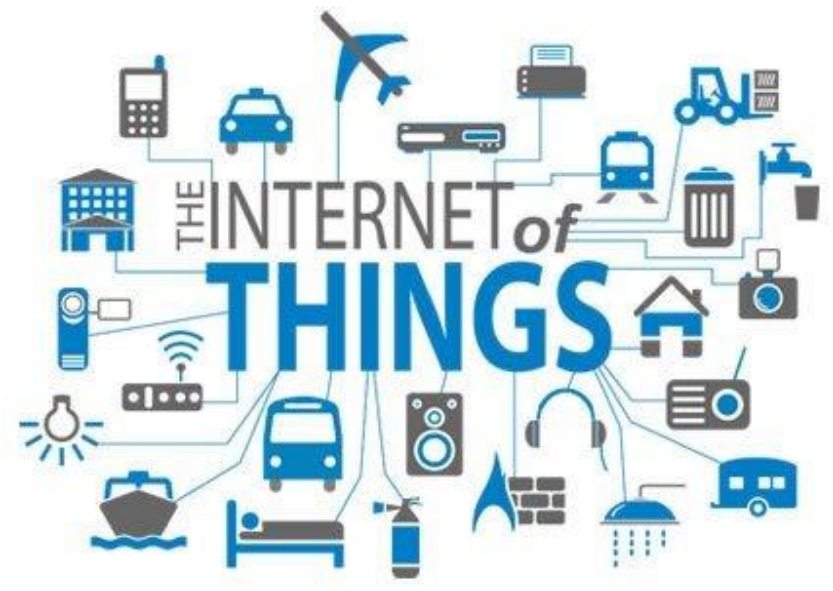

Figure 1: IoT (Source: quizlet.com)

IoT through artificial intelligence and machine learning reduces labor costs by automating jobs and processes for companies (Donepudi et al., 2020a). It reduces waste and makes service delivery more efficient. In the long run, client transactions become more transparent, and the cost of production and delivery reduces remarkably.

The IoT currently plays a very crucial role in our everyday life and remains a vital technology (Paruchuri, 2019). It would keep developing into a more advanced level as more business enterprises, and people use it. More people would get to understand the need for a connected network of the device.

\section{Edge Computing}

An edge computing system is a shared IT (Information Technology) system where customer data can be processed at the edge of the network to as close as possible to the originating source. The importance of data to modern-day businesses cannot be overemphasized. It is the base on which modern age industries strive. It provides important knowledge and makes it possible for vital business operations and processes to take place.

Currently, there are excess data, and businesses are overwhelmed with the enormous amount of data available (Ahmed et al., 2021a). However, this excess data can be gathered and categorized using Internet of Things (IoT) devices and sensors that operate in computer time from isolated locations and adverse computing surroundings anywhere on the globe and even in space.

The excess availability of data has, however, changed the ways businesses carrying out computing processes. The common technology processing networks created on a central server and traditional internet will not handle the constantly accumulating stream of real-world data (Paruchuri, 2020; Vadlamudi, 2021a).
Issues such as latency, bandwidth limitations, and unpredictable network interference can all combine to make it difficult to manage and handle huge amounts of data.

This is a challenge that businesses are trying to resolve using an edge computing system. Edge computing, in simpler terms, moves some storage portion and resources of the computer from the central database to the owner of the data itself, making it closer to the originating source. Raw data are processed and analyzed to generate data instead of being transmitted to a central data center to process and carry out an analysis (Paruchuri, 2021). This may be carried out by different sectors from retail stores to factories or even through a smart city. After analyzing data and process, insights in real-time business, predictions for equipment maintenance, and other set functions, the reports are transmitted to the central database for assessment and human supervision.

Information technology and business processes are constantly getting advanced through edge computing as it gathers data brings the data closer.

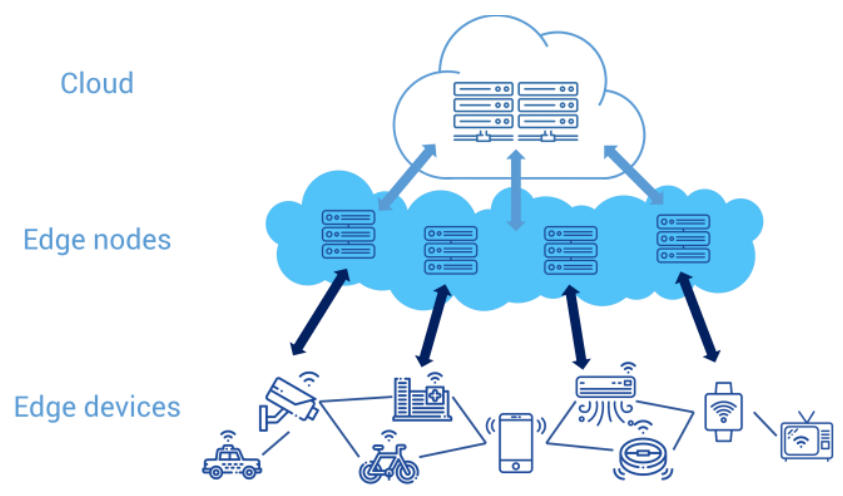

Figure 2: Edge Computing (Source: alibabacloud.com)

\section{How Edge Computing WoRKS}

Edge computing deals mainly with locations. In common business processing, data is created at a customer's endpoint, and this is the customer's device (laptop, mobile phones, and so on). Data is transmitted using wide area networks (WAN), like the internet, through the corporate local area networks. This is where storage and processing of the data by the corporation's application are carried out (Paruchuri et al., 2021). After the process has been carried out, the results are transmitted to the customer's computer (the endpoint). It remains that this process is a tested and trusted procedure for the client-server computer process for common business applications.

The volume of data produced from devices connected to the internet increases due to the growing number of internet-connected devices. As businesses utilize these data, the growth of data volume and the number of computers connected to the internet will overwhelm the common data center infrastructures. 
According to Vadlamudi, by 2021b, most businessgenerated data will not be generated on the centralized data center. The possibility of moving too much data can lead to a waste of time, and sensitive data may be disrupted. This would negatively affect the Internet, which is sometimes clogged and may crash. Information Technology designers have moved from focusing on central servers to the practical edge of the technology.

This removes the administration of storage and resource processes from the central data centers to the initial place of data. The process is straightforward. Edge computing concepts are a recent advancement in IT. It has been around as ideas in remote processing.

Placing data at the place, it was generated to be more reliable than relying on just one central data center. This idea has been used for computing in remote branch offices and other offices. Less than $30 \%$ of businesses have already enforced edge computing technology; even 55\% of them find the idea interesting.

Edge computing relocates servers and storage to the origin of data. This is done mainly by using a partial framework device to run on the local area network, gathering and processing the data locally. Computing gears are deployed in hardened or shielded enclosures to prevent the negative effects of moisture, extreme temperatures, and other conditions of the environment in most cases (Vadlamudi et al., 2021). To process data, the data stream is analyzed and normalized to find business insights. Only the outcome of the data analysis will be sent to the central data center.

Businesses' intelligence ideas may differ significantly. For instance, a business environment like retails premises where surveillance footage of the display floor could integrate with the real data from sales to find the most marketed product configuration and customer desired products. Also, predictive analytics may protect equipment maintenance and detect problems for repair before real damages, and crashes happen. There may be other scenarios that are a group with utilities like generation of electricity and treatment of water to make sure that equipment functions as it ought and keeps up with the high-quality output.

\section{Edge, Cloud, And Fog Computing}

Edge, cloud, and fog computing are closely related concepts in computing. They overlap and share great similarities. They are, however, not the same thing and should not be used interchangeably. It will be quite helpful to compare the different concepts to know the differences (Vadlamudi, 2021a).

There are different ways to know the key distinctions between edge, cloud, and fog computing. One of the easiest ways is to bring out their common idea. The common idea between all of them is that they are connected to distribute computing while being preoccupied with the physical utilization of computer processes and resource storages concerning the produced data. The difference, however, relates to the location of the storage resources.

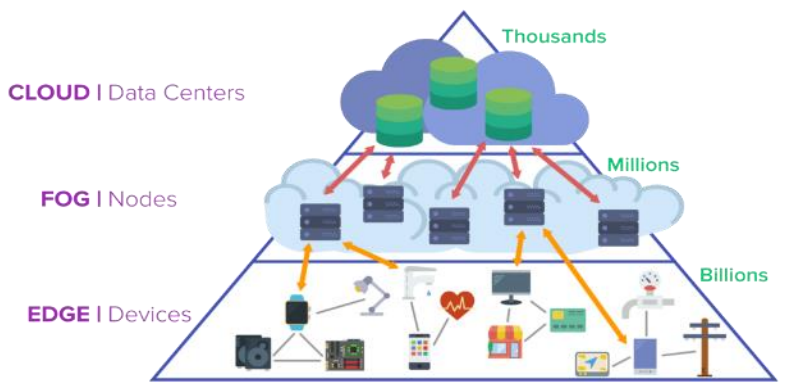

Figure 3: cloud, fog and edge computing (Source: omnisci.com)

\section{Edge computing}

Computer processes and storage resources are deployed at the origin of the data through edge computing. Through this, data processing and storage are kept in one point and used as the source of data at the network's edge. For instance, several storages and servers may be installed in small enclosures on a wind turbine to gather and process data created by detectors or sensors inside the wind turbines. Also, as an example, a good amount of storage and compute maybe be installed in railway stations to process and gather rail traffic and a great number of track sensor data. After the data has been analyzed, the outcome will then be sent to a different database for human agents to examine (Vadlamudi, $2020 \mathrm{~b}$ ). The result will also be stored with previous ones to check for patterns and greater analytics.

\section{Cloud computing}

Compute, and storage resources are deployed at a distributed global location. Cloud computing is a highly scalable and huge process. Providers of cloud services install different pre-arranged services for Internet of Things operations. It makes cloud storage the more desired centralized base for the deployment of IoT. With cloud computing, there are excess options for services and resources to carry out complicated analytics. It does not bring compute and storage close to the client. Cloud regional facilities may be thousands of miles away from where data was collected, created and internet connections are relied upon for connectivity. This is the same platform that traditional data centers are based on. Cloud computing services are normally used as alternatives to traditional data centers. Sometimes they are used side by side to compliment them. (Traditional data center). Data sources and centralized computing can be brought closer through cloud computing, not however at the network edge. 
The key difference between cloud and edge computing is that edge computing provides a system for data and sources to exist as close to each other as possible, using a network of edge devices.

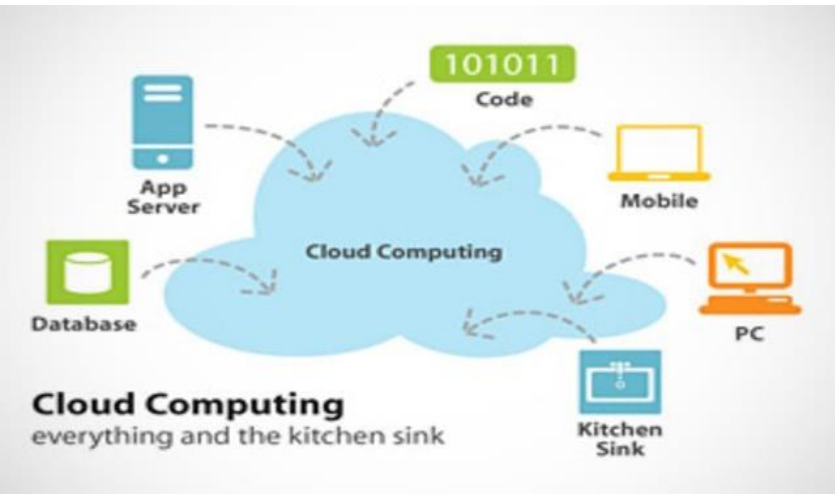

Figure 4: Cloud computing (Source: cnbc.com)

\section{Fog computing}

Edge and cloud computing are not the only options available for computing and storage resources. Fog computing can be used in place of cloud computing when the data center is not as close as needed. It can also be used as an edge due to the highly limited resource, physically distributed, and scattered nature of edge computing, making it practical. Fog computing places storage resources and computes within the data, not however at the data. Enormous amounts of internet of things and sensor data generated across extensive physical areas can be produced from a fog computing environment (Vadlamudi, 2016). These data usually too large to have an edge. Examples of such environments can are smart cities, buildings, and so on. For instance, a smart city is connected to the IoT, which allows data deployment to analyze, monitor, and enhance the city services, transportation network, and city utilities and manage urban planning. Using just an edge system would be insufficient in handling that type of workload. This is where fog computing comes in, using a series of fog nodes within the domain of the environment to gather, process, and carry out data analysis.

However, it must be understood that edge and fog computing share similar frameworks and structures, and even IT experts sometimes use them interchangeably.

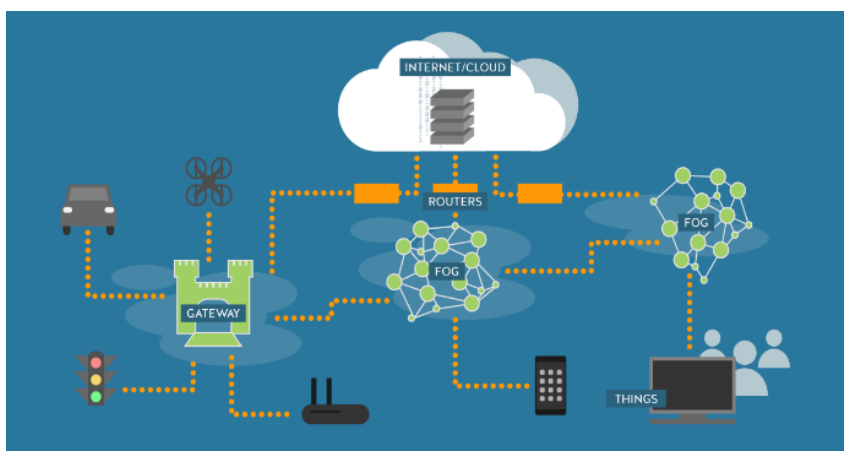

Figure 5: fog computing (Source: medium.com)

\section{Why Edge Computing Matter}

There is always a need to use a suitable framework for task computing. The framework and structure suitable for a particular task may not necessarily be suitable for every type of computing task. Edge computing is an advancement in technology that has come up with an essential and viable system that enables distributed computing to utilize storage resources and compute closer to the origin of the data ( objectively, without altering the physical location). Distributed computing models, in general, are not new. Remote and branch offices, cloud computing, and data center colocation concepts have a good history of computing performance.

Decentralizing data demands a high level of control and monitoring and can be very difficult. The level of demands can easily be overlooked during transmission from the common centralized system. The importance of edge computing has further enhanced its features which serves as a good remedy to the ensuing network issues connected with the move of a high amount of data that businesses and industries produce and use. Aside from the issue of the amount of data moved, there is also an issue of time. Computing processes depend largely on input and out of data that have become time-sensitive at an increasing level-for instance, the growth of selfdriving cars. Self-driving cars will largely depend on a traffic control system. The operations of traffic controls and cars will generate, analyze and share data in real-time. This requirement would be multiplied by numerous and large numbers of self-driving or autonomous machines, and the extent of possible issues would become obvious. This issue demands a quick response network. The use of fog and edge computing solves three main network issues: latency, Bandwidth, and congestion.

Latency issues: Latency refers to the time it takes to send and receive data between two network points. Communication over a network might happen with light speed. Sometimes they are affected by the huge distances, congestion of network, and outages which causes latency issues (delays) in data transmission in the network. These latency issues like delay affect decisionmaking processes and any analytics. The result is that system's ability to process in real-time is reduced remarkably. It can cause accidents in autonomous vehicles and lead to loss of life and properties.

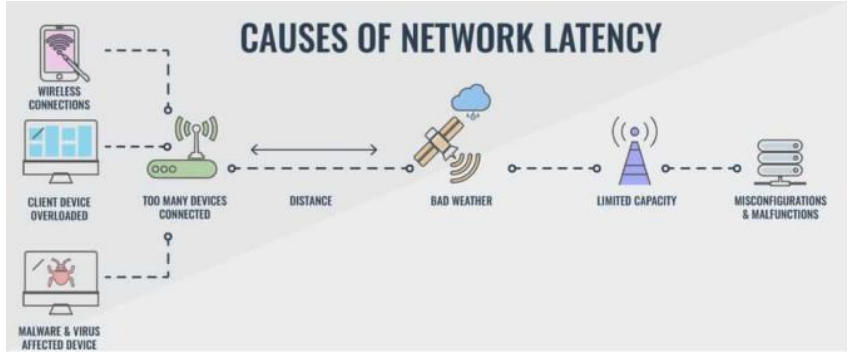

Figure 6: Latency (Source: comparitech.com) 
Bandwidth: Bandwidth refers to the volume of data a network system can process over time. It is indicated in bits per second. There limits to Bandwidth which all networks have, and it is more stringent for communications over wireless networks (Ganapathy et al., 2020). It means a limited volume of data (and devices) can be communicated over the network. Network Bandwidth can be increased to hold more data and devices; this may lead to several significant cost implications. However, increasing the limits doesn't solve all the problems.

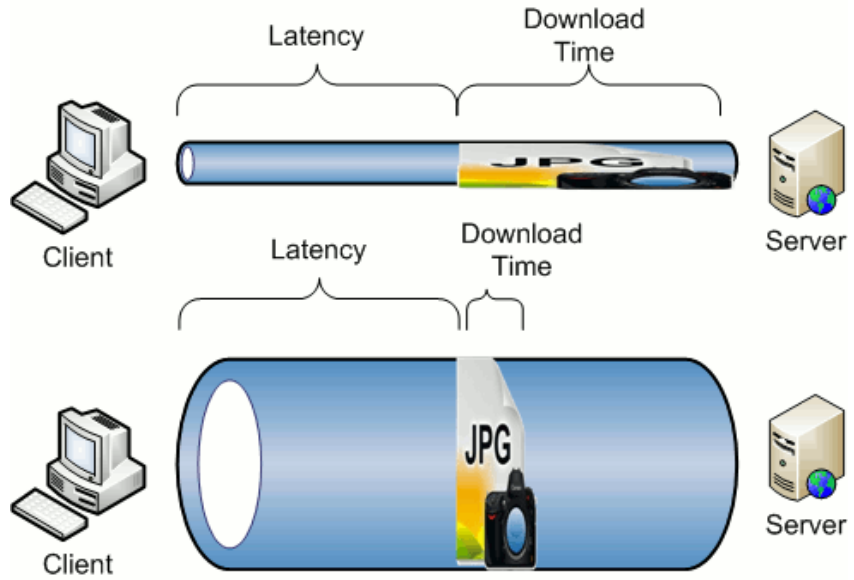

Figure 7: Bandwidth (Source: rigor.com)

Network congestion: the Internet is a world connection of "network in networks." It advanced like most technologies to deliver multipurpose data transmissions for most computer-based processes and business tasks - for example, everyday streaming and file transfers (Vadlamudi, 2020a). The amount of data being transferred increases with tens of millions of devices connected to the Internet can overpower the internet, which would cause huge degrees of congestion and lead to time-consuming retransmission of data. Network outages can worsen congestion and cut off communication entirely too several users on the internet (Ganapathy, 2019). This makes the IoT useless during outages. Edge computing uses servers and storage at the place where the data is created.

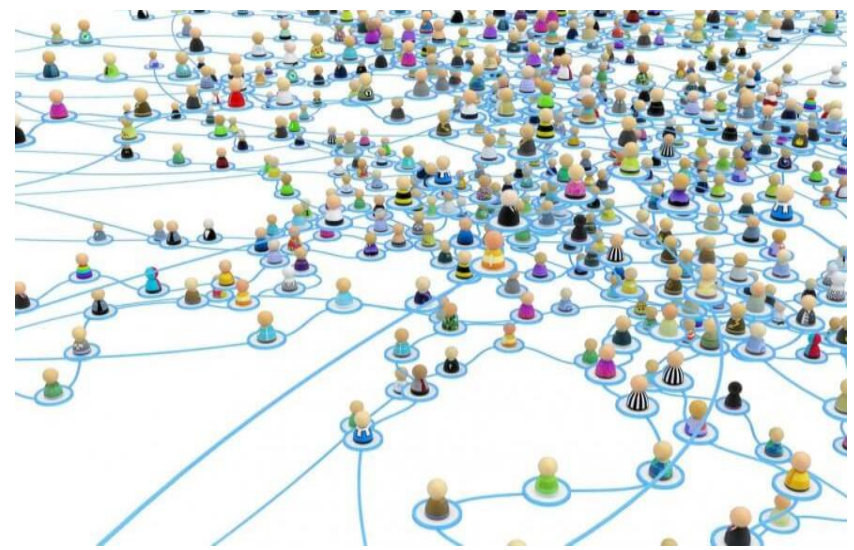

Figure 8: Network Congestion (Source: windowsreport.com)
Through this, edge computing can manage several devices using a portable and much efficient Local Area Network where enough Bandwidth can be utilized entirely by local data-generating devices eliminating congestion and latency. The raw data are gathered and protected by the local storage. In contrast, the local servers carry out important edge analytics to reduce data making it possible to finalize processes quickly before sending responses and important data to the central data center or the cloud.

\section{How Edge Computing can be Used in Different INDUSTRIES}

In administration, data analysis, filtering, processing are carried out by edge computing techniques at the edge of the network or close to it. Edge computing is an efficient way of utilizing data that cannot be transmitted initially to a centralized network storage location. The huge volume of the data makes it quite expensive and technologically ineffective. It might also be based on laws, as some laws restrict certain types of data movement.

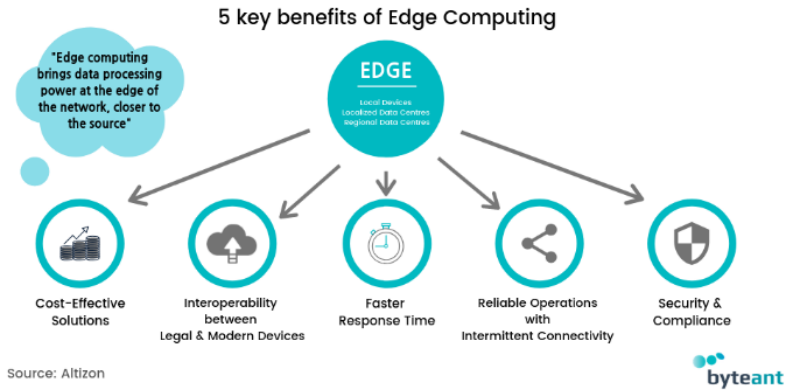

Figure 9: benefits of edge computing (Source: byteant.com)

From the following several uses of edge computing in different fields and industries. Some example are;

Manufacturing: edge computing could be used in the manufacturing industry to monitor and manage manufacturing processes and enable real-time analytics. Also, machine learning and deep learning are used at the edge of the network to detect production faults and enhance the quality of product manufacturing. Edge computing enables the implementation of sensors throughout manufacturing plants and provides insights into how components of products are stored (including the duration of they remain in stock) and assembled. Accurate and faster business decisions regarding the manufacturing structure and factory operations can be taken by manufacturers using edge computing.

Farming: imagine a farm business that tries to grow crops in an enclosed area without soil, sunlight, or pesticides. This process only rates $40 \%$ compared to the natural farming process. Sensors can keep track of the density of nutrients, water use and deduce optimal harvest. The gathered data is analyzed to learn and 
understand the impacts of environmental factors. It would help provide continuous data for improvements on algorithms for growing crops and guarantee crops are harvested at their prime.

Network optimization: through measuring users' performance across the Internet, edge computing ensures that network performance is enhanced and optimized (Donepudi et al., 2020b). Also, edge computing uses an analytics process to find out the best and dependable low latency network stream for specific user traffic. In summary, edge computing is utilized to control traffic across networks for the best time-sensitive performance of traffic.

Workplace safety: Through a combination of data analysis from onsite surveillance, data from sensor devices like employee safety devices and other sensors, edge computing can help businesses manage workplace conditions and enable better conditions to enhance the safety of employees. It can also be used to ensure employees act in line with safety policies, especially in remote workplaces or extremely dangerous places like oil rigs and construction sites.

Healthcare improvement: the amount of data gathered from the healthcare sector has greatly increased as patient data are collected from different sensors, devices, and health appliances. The huge data volume needs the use of edge computing, through the application of machine learning and automation, to process data and detect problems for clinicians to take the necessary actions on time to help patients.

Transportation: Research has shown that about 5 to 20 terabytes of data are generated by autonomous vehicles per day (Ahmed et al., 2021b). Data on other vehicles, location, vehicle condition, location, and road and traffic conditions are collected by autonomous vehicles. These data can be collated and analyzed in real-time while the vehicle is still moving. Autonomous vehicle needs substantial onboard computer processing, and they become edges.

Retail businesses: Businesses generate a high volume of data from sales, stock trading, surveillance, and other real-time business information. Edge computing can be utilized in analyzing the different data and detect opportunities in businesses. For example, sales can be predicted, and client orders can be optimized effectively. Edge computing can be effective in dealing with the drastic retail business changes in the local environment and solve local processing issues at each.

\section{Possibilities Of Edge Computing AND IoT}

The growth and application of different technological innovations to improve performance and abilities in modern times have expanded remarkably. Edge computing is part of the technological growth which evolved to optimize processes and performance. The trend of edge computing expansion and growth has seen a potential rise and application in different industries worldwide by 2028. Currently, edge computing is used for specific situations. The growth and advancement in technology would see it become universal and break away from normal internet utilization. This would increase its features and new potential application cases.

The increase can be detected from the increase of compute, network and storage device products designed precisely for edge computing (Amin \& Vadlamudi, 2021). Also, an increase in multi-vendor relationships will allow for the best product interoperability and edge flexibility-for instance, Verizon and AWS partnership, leading to increased edge connectivity.

Wi-Fi 6 and 5G, which are technologies that allow communications over a wireless network, will influence the use and deployment of edge computing in the future. It will allow automation and visualization skills that have not been examined, for instance, fully autonomous vehicles and a large volume of data transfer.

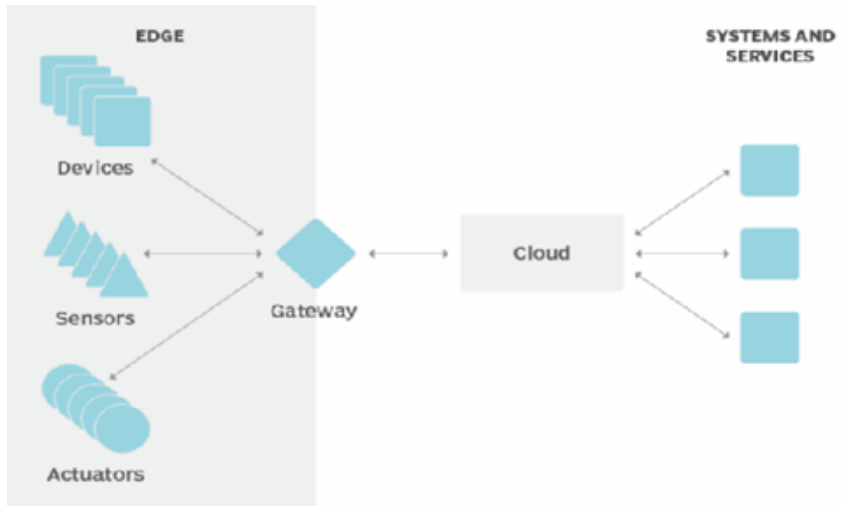

Figure 10: IoT gateways (Source: internetofthingsagenda.techtarget.com)

The rise of the internet of things and the unexpected increase in data produced from IoT-connected devices greatly influenced the popularity of edge computing. However, the Internet of Things technology is still at the infancy level; its growth and development will greatly affect the development of edge computing. Micro modular data centers (MMDCs) development is one of the future alternatives. MMDCs are practically data hubs in boxes that are a complete data center are put in a movable little system and can be used closer to data. It brings computing as close to data as possible without establishing the edge at the data entirely.

\section{CONCLUSION}

The Internet of Things functions today across a wide range of networks and applications without edge computing. However, with the increase in devices connected to the 
IoT, edge computing would become important in enabling the IoT to optimize functions. This can be detected from the increase in new application cases, which will result in higher latency and bandwidth, and security needs. There are, however, challenges and undiscovered cases use, which would help discover the nature of the IoT and edge computing would play in the future. Amongst these are technical issues surrounding making sure there is physical security for edge infrastructure when data is being stored outside the highly secured environment of data centers. Other technical aspects many programs try to solve are understanding flexibility issues and reducing device control and management. In the future, accessing edge would cost premium charges compared to a centralized cloud. Connections between IoT devices that want to connect to edge devices would ensue several enormous benefits to justify the premium fee.

\section{REFERENCES}

Ahmed, A. A. A., Aljarbouh, A., Donepudi, P. K., \& Choi, M. S. (2021a). Detecting Fake News using Machine Learning: A Systematic Literature Review. Psychology and Education, 58(1), 1932-1939. https://zenodo.org/record/4494366. https://doi.org/10.5281/zenodo.4494366

Ahmed, A. A. A.; Paruchuri, H.; Vadlamudi, S.; \& Ganapathy, A. (2021b). Cryptography in Financial Markets: Potential Channels for Future Financial Stability. Academy of Accounting and Financial Studies Journal, 25(4), 1-9. https://doi.org/10.5281/zenodo.4774829

Amin, R., \& Vadlamudi, S. (2021). Opportunities and Challenges of Data Migration in Cloud. Engineering International, 9(1), 41-50. https://doi.org/10.18034/ei.v9i1.529

Azad, M. M., Ganapathy, A., Vadlamudi, S., Paruchuri, H. (2021). Medical Diagnosis using Deep Learning Techniques: A Research Survey. Annals of the Romanian Society for Cell Biology, 25(6), 5591-5600. Retrieved from https://www.annalsofrscb.ro/index.php/journal/article/ view $/ 6577$

Donepudi, P. K., Ahmed, A. A. A., Hossain, M. A., \& Maria, P. (2020a). Perceptions of RAIA Introduction by Employees on Employability and Work Satisfaction in the Modern Agriculture Sector. International Journal of Modern Agriculture, 9(4), 486-497. https://doi.org/10.5281/zenodo.4428205

Donepudi, P. K., Banu, M. H., Khan, W., Neogy, T. K., Asadullah, ABM., \& Ahmed, A. A. A. (2020b). Artificial Intelligence and Machine Learning in Treasury Management: A Systematic Literature Review. International Journal of Management, 11(11), 13-22. https://doi.org/10.5281/zenodo.4247297
Ganapathy, A. (2019). Mobile Remote Content Feed Editing in Content Management System. Engineering International, 7(2), 85-94. https://doi.org/10.18034/ei.v7i2.545

Ganapathy, A., Redwanuzzaman, M., Rahaman, M. M., \& Khan, W. (2020). Artificial Intelligence Driven Crypto Currencies. Global Disclosure of Economics and Business, 9(2), 107-118. https://doi.org/10.18034/gdeb.v9i2.557

Paruchuri, H. (2019). Market Segmentation, Targeting, and Positioning Using Machine Learning. Asian Journal of Applied Science and Engineering, 8(1), 7-14. Retrieved from https://journals.abc.us.org/index.php/ajase/article/view $\underline{1193}$

Paruchuri, H. (2020). The Impact of Machine Learning on the Future of Insurance Industry. American Journal of Trade and Policy, 7(3), 85-90. https://doi.org/10.18034/ajtp.v7i3.537

Paruchuri, H. (2021). Conceptualization of Machine Learning in Economic Forecasting. Asian Business Review, 11(1), 51-58. https://doi.org/10.18034/abr.v11i1.532

Paruchuri, H.; Vadlamudi, S.; Ahmed, A. A. A.; Eid, W.; Donepudi, P. K. (2021). Product Reviews Sentiment Analysis using Machine Learning: A Systematic Literature Review. Turkish Journal of Physiotherapy and Rehabilitation, 23(2), 2362-2368 https://turkjphysiotherrehabil.org/pub/pdf/322/32-2316.pdf

Vadlamudi, S. (2016). What Impact does Internet of Things have on Project Management in Project based Firms?. Asian Business Review, 6(3), 179-186. https:/ / doi.org/10.18034/abr.v6i3.520

Vadlamudi, S. (2020a). Internet of Things (IoT) in Agriculture: The Idea of Making the Fields Talk. Engineering International, 8(2), 87-100. https://doi.org/10.18034/ei.v8i2.522

Vadlamudi, S. (2020b). The Impacts of Machine Learning in Financial Crisis Prediction. Asian Business Review, 10(3), 171176. https://doi.org/10.18034/abr.v10i3.528

Vadlamudi, S. (2021a). The Economics of Internet of Things: An Information Market System. Asian Business Review, 11(1), 3540. https://doi.org/10.18034/abr.v11i1.523

Vadlamudi, S. (2021b). The Internet of Things (IoT) and Social Interaction: Influence of Source Attribution and Human Specialization. Engineering International, 9(1), 17-28. https://doi.org/10.18034/ei.v9i1.526

Vadlamudi, S.; Paruchuri, H.; Ahmed, A. A. A.; Hossain, M. S.; \& Donepudi, P. K. (2021). Rethinking Food Sufficiency with Smart Agriculture using Internet of Things. Turkish Journal of Computer and Mathematics Education, 12(9), 2541-2551. https://turcomat.org/index.php/turkbilmat/article/view $\angle 3738$

$$
--0--
$$




\section{How to Cite This Article}

Ganapathy, A. (2021). Edge Computing: Utilization of the Internet of Things for Time-Sensitive Data Processing. Asian Business Review, 11(2), 59-66. https://doi.org/10.18034/abr.v11i2.547 